\title{
Development of Hemolytic Anemia in a Nivolumab-Treated Patient with Refractory Metastatic Squamous Cell Skin Cancer and Chronic Lymphatic Leukemia
}

\author{
K.S. Schwab ${ }^{a} \quad$ A. Heine ${ }^{a} \quad$ T. Weimann ${ }^{b} \quad$ G. Kristiansen ${ }^{c} \quad$ P. Brossart ${ }^{a}$ \\ ${ }^{a}$ Department of Internal Medicine 3, University Hospital of Bonn, Bonn, Germany; \\ ${ }^{b}$ Betaklinik Bonn, Bonn, Germany; ' Institute of Pathology, University Hospital of Bonn, \\ Bonn, Germany
}

\section{Keywords}

Nivolumab $\cdot$ Squamous cell carcinoma $\cdot$ Hemolysis

\begin{abstract}
Management of patients with metastatic squamous cell skin cancer, refractory to initial therapy with standard chemotherapy and radiation protocols, remains difficult with poor overall prognosis and limited therapeutic options. Recently, promising response rates with nivolumab, a programmed death receptor-1-blocking antibody, in squamous cancer of the head and neck have been demonstrated. Considering the similar histological patterns of squamous cell cancer of the skin and squamous cell cancer of the head and neck, we assumed that nivolumab could also be effective in our patients with refractory metastatic squamous cell cancer of the skin. So far, there have been no clinical data on the therapeutic efficacy of nivolumab in squamous cell skin cancer. We here present a case of a patient with metastatic squamous cell skin cancer refractory to previous therapies, who showed a good response to nivolumab over a period of 5 months, but developed a serious hemolytic crisis under nivolumab treatment after eight applications.




\section{Introduction}

Cutaneous squamous cell carcinoma (SCC) is the second most common type of nonmelanoma skin cancer [1]. Management of patients with metastatic squamous cell skin cancer, refractory to initial therapy with standard chemotherapy and radiation protocols, remains difficult with poor overall prognosis and limited therapeutic options.

Chemotherapy is one of the options for the treatment of inoperable cutaneous SCC. Other systemic treatments are not well studied. Extrapolations for skin SCC therapy have been made, with varying degrees of success, from regimens utilized in head and neck SCCs, but prospective trials are lacking. Active agents include platinum compounds, 5-fluorouracil, or biologic response modifiers such as interferon-alpha or retinoic acids [2-6]. Nevertheless, success rates and progression-free survival rates are low.

Recently, promising response rates with nivolumab, a programmed death receptor-1 (PD-1)-blocking antibody, in squamous cell cancer of the head and neck have been demonstrated [7]. Nivolumab has been approved by the Food and Drug Administration for the treatment of squamous non-small cell lung cancer. Considering the similar histological patterns of squamous cell cancer of the skin and squamous cell cancer of the head and neck, we assumed that nivolumab could also be effective in patients with refractory metastatic squamous cell cancer of the skin.

\section{Case}

We present the history of an 82-year-old man with chronic lymphatic leukemia (B-CLL), diagnosed in 2004 who received rituximab, fludarabine and cyclophosphamide for four cycles between May 2010 and October 2010 as first-line therapy. The past medical history showed arterial hypertension and prostate cancer treated with radical prostatectomy in 2005.

In July 2014, an SCC of the skin located in the scalp was diagnosed and completely resected without any signs of metastases. At that time, there was no sign of progressive B-CLL. In January 2015, the patient presented with progressive lymphadenopathy, night sweats, fever and weight loss and a worsening of peripheral blood count with a decrease in hemoglobin (from 11 to $9 \mathrm{~g} / \mathrm{dl}$ ) and a reduction of thrombocytes (from 60 to $20 \times 10^{9} / \mathrm{l}$ ). Further examinations revealed a progression of the B-CLL with generalized lymphadenopathy and an increase in monoclonal B-CLL population (up to 65\%) in peripheral blood detected by FACS. Therefore, salvage therapy with ibrutinib was started in January 2015.

After an initially very good response to ibrutinib with fast significant lymph node regression and improvement of the peripheral blood count, in February 2015, he presented with progressive painful cervical swelling and numbness of the right side of his face. At that time, the B-CLL was in stable remission, and therapy with ibrutinib was stopped immediately. An MRI scan revealed a progressive cervical tumor. A lymph node biopsy showed an infiltration of a keratinizing, moderately differentiated squamous cell cancer of the skin due to a metastatic manifestation of the squamous cell cancer of the scalp diagnosed in July 2014. In February, first-line treatment with primary radiochemotherapy (cisplatin $20 \mathrm{mg} / \mathrm{m}^{2}$ days 15 and radiation with $60 \mathrm{~Gy}$ ) was initiated. He received a total of three cycles until April 2015. There were no signs of metastases at the end of therapy. However, the primary tumor, located retroauricular, was not resectable. In August 2015, a CT scan showed progressive disease with new tumor manifestations in the lung, cervical lymph nodes and mediastinum. Subse- 


\section{Case Reports in Oncology}

quently, in August 2015, a second-line chemotherapy consisting of cetuximab and docetaxel was initiated.

In September 2015, a CT scan revealed a refractory disease with significant tumor progression after two cycles of chemotherapy. Further analysis detected positive PD-1 expression of the tumor (fig. 1). In accordance with the patient's insurance, a therapy with nivolumab was started. From October 2015 until January 2016, nivolumab was administered at a dose of $3 \mathrm{mg} / \mathrm{kg}$ intravenously every 2 weeks. Therapy was well tolerated and side effects were unremarkable except for generalized muscle weakness and fatigue. In particular, there were no severe infections or signs of autoimmune disease. Routine follow-up laboratory parameters remained mostly normal. During nivolumab therapy, no changes in immune cells were detected with a reduced, but stable T-cell count (CD4+ and CD8+ $<200 / \mu \mathrm{l}$ ) before, during and after treatment with nivolumab.

After four applications of nivolumab, a CT scan was performed that demonstrated a significant response with marked size regression of the main cervical tumor manifestation and of the pulmonal manifestations. Abdominal lymph nodes, probably due to the B-CLL showed stable disease. Therefore, therapy with nivolumab was continued and was well tolerated. The initial weakness improved after the third administration. In February 2016, after eight administrations of nivolumab, the patient presented with a rapid progressive decrease of hemoglobin. At that point, there was no sign of progressive CLL as lymph node manifestations of CLL were stable with a constant proportion of monoclonal B-lymphocytes due to BCLL in peripheral blood detected in FACS analysis.

Further laboratory examinations confirmed the diagnosis of acute hemolysis with detection of irregular antibodies. Detailed diagnostics detected warm antibodies with positive direct agglutinin test of anti-IgG and anti-C3.

Consequently, nivolumab was terminated and treatment with prednisolone $80 \mathrm{mg} / \mathrm{day}$ was started. After 2 weeks, hemolysis was stopped and peripheral blood count stabilized. At re-evaluation in February 2016, after a total of eight administrations of nivolumab, CT imaging showed a good response with further regression of the cervical, thoracal and mediastinal tumor manifestations (fig. 2a, b). Due to the observed hemolysis, a progressive splenomegaly was diagnosed. One month after termination of nivolumab treatment, the patient presented in good physical condition with recovered peripheral blood count and no signs of hemolysis or tumor progression. Finally under third-line therapy with nivolumab, squamous cell skin cancer showed a satisfying response for a duration of at least 5 months (from October 2015 until March 2016).

\section{Discussion}

We here present a case of a patient with metastatic squamous cell skin cancer refractory to previous therapies, who showed a good response to nivolumab over a period of 5 months, but developed a serious hemolytic crisis under nivolumab treatment after eight applications. Patients suffering from metastatic squamous cell skin cancer refractory to initial therapy carry an exceptionally poor overall prognosis, and therapeutic options are limited. Apart from a recently published case report showing efficacy of nivolumab in squamous cell skin cancer [8], there have been no clinical data on the therapeutic efficacy of the PD-1 antibody nivolumab in this setting so far.

In patients with recurrent or metastatic platinum-refractory SCC of the head and neck, the pivotal phase 3 trial CheckMate-141 demonstrated superior overall survival with 


\section{Case Reports in Oncology}

nivolumab (Opdivo) [7]. CheckMate-141 is an open-label study that evaluated nivolumab compared with the investigator's choice of therapy. A total of 361 participants with SCC of the head and neck who experienced disease progression on or within 6 months of platinum therapy were randomly assigned 2:1 to receive nivolumab $3 \mathrm{mg} / \mathrm{kg}$ intravenously every 2 weeks, or cetuximab, methotrexate, or docetaxel until disease progression or unacceptable toxicity.

Considering the similar histological pattern of squamous cell cancer of the skin and that of the head and neck, nivolumab is likely to be equally effective in squamous cancer of the skin. As shown in previous studies, most of the regimens used in the treatment of squamous cancer of the head and neck also show significant efficacy in squamous cancer of the skin [912].

Our patient received a total of eight administrations of nivolumab. Although he had been refractory to platinum-based chemotherapy as well as to second-line therapy with docetaxel and cetuximab, he showed a significant response to nivolumab with marked regression of the main tumor lesions for at least 5 months.

Safety, rash, musculoskeletal pain, constipation, diarrhea, and decreased appetite are frequently reported with single-agent nivolumab treatment. In our patient, side effects were moderate and therapy was well tolerated.

After eight administrations of nivolumab, acute autoimmune hemolysis occurred in our patient. This could be caused either by nivolumab or by any other drug administered at that time. Recently, Kong et al. [13] reported a case of hemolysis under nivolumab in a patient with metastatic melanoma. But in this case, the patient had a history of multiple red cell alloantibodies and a positive direct antiglobulin test before the start of nivolumab therapy. In contrast, in our patient, there has never been evidence of hemolysis or red cell antibodies before the start of nivolumab.

The largest series of cases of drug-induced immune hemolytic anemia (IHA) was reported in the Berlin Case-Control Surveillance Study, which included 124 cases in which IHA developed in an outpatient setting and 731 controls [14]. Significantly increased odds ratios for the development of IHA were observed for beta-lactam antibiotics, cotrimoxazole, ciprofloxacin, fludarabine, lorazepam, and diclofenac. A 2015 report of 73 patients with druginduced immune hemolysis identified the following as major causative agents: diclofenac, piperacillin, ceftriaxone, oxaliplatin [15]. At the time of hemolysis, none of the drugs suspected to cause autoimmune hemolysis had been administered.

Alternatively, autoimmune hemolysis could be due to the B-CLL. However, at that point, there was no sign of progressive CLL in our patient. Lymph node manifestations of CLL were stable with a constant proportion of monoclonal B-lymphocytes (40\%) due to B-CLL in peripheral blood detected in FACS analysis. Moreover, hemolysis had never occurred before in our patient, despite the long history of B-CLL diagnosed in 2004. However, the origin of hemolysis in this case remains unknown.

This case report shows a promising response to nivolumab of metastatic squamous skin cancer refractory to previous treatment regimens. Despite the serious hemolysis that occurred and might be associated with nivolumab, side effects were moderate even in a patient of advanced age with concomitant diseases. Consequently, nivolumab should be considered as a promising treatment option in patients with squamous cell skin cancer, even in primary tumors refractory to previous regimens. Further studies will be needed to evaluate the efficacy and safety of nivolumab in squamous cell skin cancer. 


\section{Statement of Ethics}

No interventions were performed as part of this case report. Neither approval by an Ethics Committee nor patient consent is necessary in the state of Northrhine-Westphalia, Germany.

\section{Disclosure Statement}

None.

\section{References}

1 DeConti RC: Chemotherapy of squamous cell carcinoma of the skin. Semin Oncol 2012;39:145-149.

2 Samarasinghe V, Madan V, Lear JT: Management of high-risk squamous cell carcinoma of the skin. Expert Rev Anticancer Ther 2011;11:763-769.

3 Brewster AM, Lee JJ, Clayman GL, et al: Randomized trial of adjuvant 13-cis-retinoic acid and interferon alfa for patients with aggressive skin squamous cell carcinoma. J Clin Oncol 2007;25:1974-1978.

4 Lippman SM, Parkinson DR, Itri LM, et al: 13-cis-retinoic acid and interferon alpha-2a: effective combination therapy for advanced squamous cell carcinoma of the skin. J Natl Cancer Inst 1992;84:235-241.

5 Shin DM, Glisson BS, Khuri FR, et al: Phase II and biologic study of interferon alfa, retinoic acid, and cisplatin in advanced squamous skin cancer. J Clin Oncol 2002;20:364-370.

6 Cartei G, Cartei F, Interlandi G, et al: Oral 5-fluorouracil in squamous cell carcinoma of the skin in the aged. J Clin Oncol 2000;23:181-184.

7 Princeton NJ: CheckMate-141, a pivotal phase 3 Opdivo (nivolumab) head and neck cancer trial, stopped early (news release). Bristol-Myers Squibb, January 28, 2016. http://news.bms.com/pressrelease/checkmate-141-pivotal-phase-3-opdivo-nivolumab-head-and-neck-cancer-trial-stopped-earl. Accessed January 29, 2016.

-8 Borradori L, Sutton B, Shayesteh P, Daniels GA: Rescue therapy with anti-programmed cell death protein 1 inhibitors (PD-1) of advanced cutaneous squamous cell carcinoma and basosquamous carcinoma: preliminary experience in 5 cases. Br J Dermatol 2016, DOI: 10.1111/bjd.14642.

-9 Stratigos A, Garbe C, Lebbe C, Malvehy J, del Marmol V, Pehamberger H, Peris K, Becker JC, Zalaudek I, Saiag P, Middleton MR, Bastholt L, Testori A, Grob JJ; European Dermatology Forum (EDF); European Association of Dermato-Oncology (EADO); European Organization for Research and Treatment of Cancer (EORTC): Diagnosis and treatment of invasive squamous cell carcinoma of the skin: European consensus-based interdisciplinary guideline. Eur J Cancer 2015;51:1989-2007.

10 Swiecicki PL, Malloy KM, Worden FP: Advanced oropharyngeal squamous cell carcinoma: pathogenesis, treatment, and novel therapeutic approaches. World J Clin Oncol 2016;7:15-26.

-11 Sacco AG, Cohen EE: Current treatment options for recurrent or metastatic head and neck squamous cell carcinoma. J Clin Oncol 2015;33:3305-3313.

-12 Cranmer LD, Engelhardt C, Morgan SS: Treatment of unresectable and metastatic cutaneous squamous cell carcinoma. Oncologist 2010;15:1320-1328.

13 Kong BY, Micklethwaite KP, Swaminathan S, Kefford RF, Carlino MS: Autoimmune hemolytic anemia induced by anti-PD-1 therapy in metastatic melanoma. Melanoma Res 2016;26:202-204.

14 Garbe E, Andersohn F, Bronder E, et al: Drug induced immune haemolytic anaemia in the Berlin CaseControl Surveillance Study. Br J Haematol 2011;154:644.

15 Mayer B, Bartolmäs T, Yürek S, Salama A: Variability of findings in drug-induced immune haemolytic anaemia: experience over 20 years in a single centre. Transfus Med Hemother 2015;42:333. 


\section{Case Reports in Oncology}

\begin{tabular}{l|l}
\hline Case Rep Oncol 2016;9:373-378 \\
\hline $10.1159 / 000447508$ & $\begin{array}{l}\text { @ 2016 The Author(s). Published by S. Karger AG, Basel } \\
\text { www.karger.com/cro }\end{array}$ \\
\hline
\end{tabular}

Schwab et al: Development of Hemolytic Anemia in a Nivolumab-Treated Patient with Refractory Metastatic Squamous Cell Skin Cancer and Chronic Lymphatic Leukemia

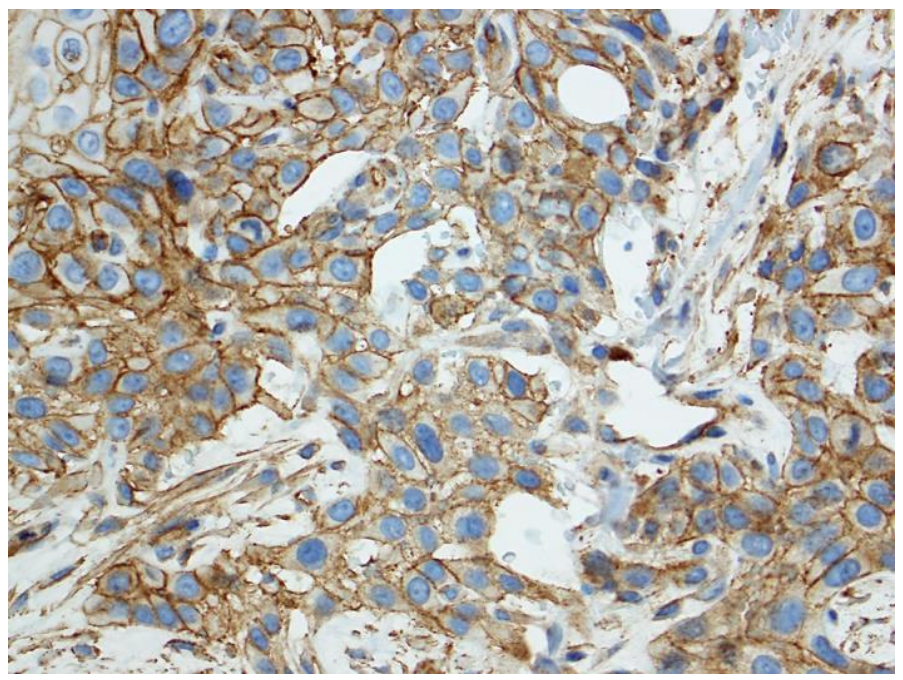

Fig. 1. Positive PD-1 expression of the tumor.
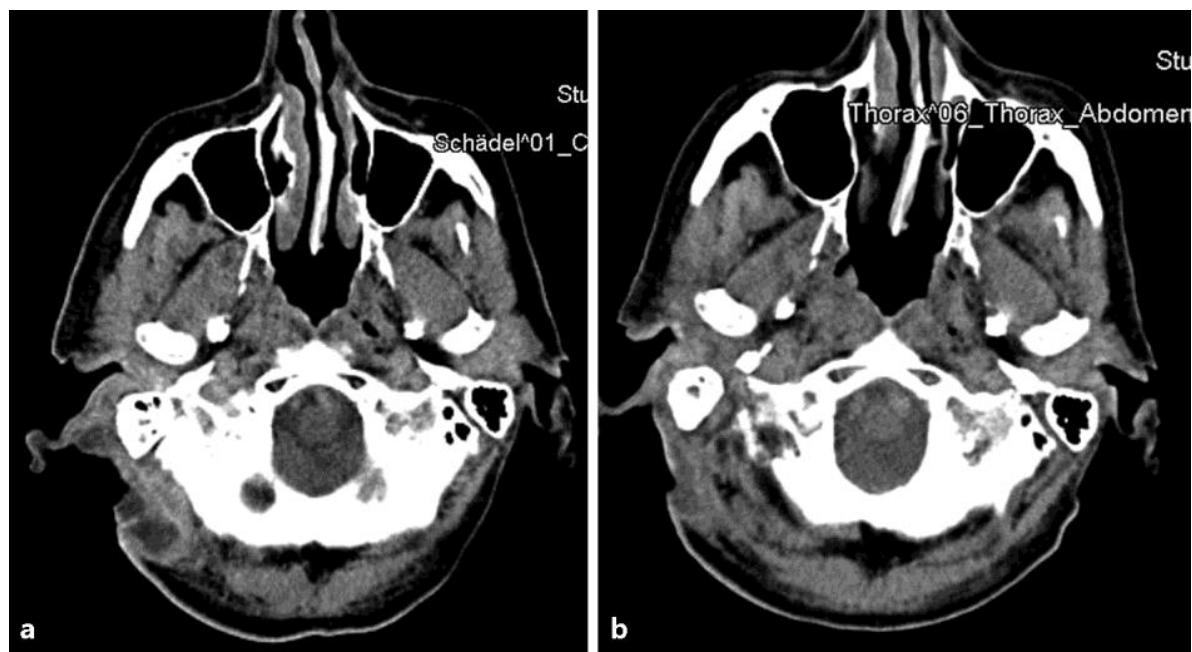

Fig. 2. a, b Good response to nivolumab with further regression of the cervical, thoracal and mediastinal tumor manifestations. 